\title{
Novel approaches to assess the quality of fertility data stored in dairy herd management software
}

\author{
K. Hermans, ${ }^{* 1}$ W. Waegeman,† G. Opsomer, ${ }^{*}$ B. Van Ranst, ${ }^{*}$ J. De Koster, ${ }^{*}$ M. Van Eetvelde, ${ }^{*}$ and M. Hostens ${ }^{*}$ \\ *Faculty of Veterinary Medicine, Department of Reproduction, Obstetrics and Herd Health, Ghent University, Salisburylaan 133,9820 Merelbeke, \\ Belgium \\ †Faculty of Bioscience Engineering, Department of Mathematical Modelling, Statistics and Bioinformatics, Ghent University, Coupure Links 653, \\ 9000 Ghent, Belgium
}

\begin{abstract}
Scientific journals and popular press magazines are littered with articles in which the authors use data from dairy herd management software. Almost none of such papers include data cleaning and data quality assessment in their study design despite this being a very critical step during data mining. This paper presents 2 novel data cleaning methods that permit identification of animals with good and bad data quality. The first method is a deterministic or rule-based data cleaning method. Reproduction and mutation or life-changing events such as birth and death were converted to a symbolic (alphabetical letter) representation and split into triplets (3-letter code). The triplets were manually labeled as physiologically correct, suspicious, or impossible. The deterministic data cleaning method was applied to assess the quality of data stored in dairy herd management from 26 farms enrolled in the herd health management program from the Faculty of Veterinary Medicine Ghent University, Belgium. In total, 150,443 triplets were created, $65.4 \%$ were labeled as correct, $17.4 \%$ as suspicious, and $17.2 \%$ as impossible. The second method, a probabilistic method, uses a machine learning algorithm (random forests) to predict the correctness of fertility and mutation events in an early stage of data cleaning. The prediction accuracy of the random forests algorithm was compared with a classical linear statistical method (penalized logistic regression), outperforming the latter substantially, with a superior receiver operating characteristic curve and a higher accuracy (89 vs. 72\%). From those results, we conclude that the triplet method can be used to assess the quality of reproduction data stored in dairy herd management software and that a machine learning technique such as random forests is capable of predicting the correctness of fertility data.
\end{abstract}

Received August 19, 2016.

Accepted January 4, 2017.

${ }^{1}$ Corresponding author: kristofhermans@bovinet.be
Key words: dairy reproduction, data quality, dairy herd management software, random forests

\section{INTRODUCTION}

Researchers often use data stored in dairy herd management software to facilitate the collection of fertility data (Caraviello et al., 2006; Zwald et al., 2006; Shahinfar et al., 2014). These are so-called secondary data sources, referring to the fact that the data are collected by someone other than the user (i.e., the researcher) and not specifically collected for research purposes. Conclusions based on data from dairy herd management software are regularly published in scientific journals (Zwald et al., 2004; Caraviello et al., 2006; Wenz and Giebel, 2012) without proper data quality assessment or data cleaning (Harpe, 2009). Despite the increasing importance of data quality (Wang and Strong, 1996; Arts et al., 2002) and the rich theoretical and practical contributions in all aspects of data cleaning (Ballou and Pazer, 1985; Wand and Wang, 1996; Pipino et al., 2002), no single end-to-end off-the-shelf solution is available to automate the detection of incorrect data. Often a significant portion of the cleaning work has to be done manually or by low-level programs (Rahm and Do, 2000), making data quality assessment an expensive and time-consuming process (Wang et al., 1995; Haug et al., 2011). In addition, the ability to gather data via handheld computers, as well as more complex data capturing systems working in tandem with technologies such as voluntary milking systems and heat detection aids, has outpaced the speed and cost of convenient data quality assessment.

The first objective of the present study was to develop a deterministic or rule-based data cleaning method that is easy to understand and quick to implement. A novel method for screening physiologically plausible or implausible sequences of reproduction events was introduced. The time series events for every animal were converted to a symbolic representation and split into triplets (3-letter code). To this end, triplets were 
manually labeled as physiological correct, suspicious, or impossible. As such it becomes possible to determine whether or not data from an individual cow are complete and correct to serve as input for statistical analysis.

The second objective of the present study was to develop a probabilistic or prediction-based data cleaning method based on cow- and farm-related variables. The probabilistic data cleaning method could be used to preselect cow records with a high probability of being correct at the time of data extraction and in the future (early phase data assessment). Hence, time and cost needed for deterministic data cleaning could decline (Haug et al., 2011).

To do so, we adopted a machine learning methodology, random forests (RF; Breiman, 2001), by searching for statistical relationships between data correctness and certain predictor variables that are characteristic for an individual cow. To this end, we first defined what we consider as correct and incorrect data records. This definition was used to manually label the records from dairy cows on 26 farms. The database was split into a training set, which served as input to train the RF, and a testing data set, which served to validate the prediction performance of the RF.

In our experimental results, the detection performance of RF (Breiman, 2001) is compared with a classical linear statistical method (penalized logistic regression, PLR; Loeffler et al., 1999; Fourichon et al., 2000; López-Gatius et al., 2005). Machine learning algorithms are able to accommodate for complex nonlinear relationships within data; for that reason, their prediction capabilities often outperform classical statistical methods (Lim et al., 2000; Loh, 2011). Additionally, variables affecting the quality of reproductive data from dairy cows are identified. These variables could be of interest for including or excluding farms and cows for government, disease control, or research purposes.

\section{MATERIALS AND METHODS}

\section{Data Extraction}

Twenty-six farms enrolled in the herd health management program from the Faculty of Veterinary Medicine, Ghent University, Belgium, and those possessing a dairy management software program, were included in the study. Backup files from the herd management software used on these farms were extracted by the Dairy Data Warehouse (2016; DDW) and stored without manipulation of the data. Reproduction and mutation or life-changing events like birth, purchase, sale, or death data were obtained from DDW via a web appli- cation programming interface. Reproduction events encountered in the present study were heat, insemination, positive pregnancy check, negative pregnancy check, do not breed, and abortion. Natural mating, AI, and embryo transfer were all classified as an insemination event. No other data than mutation and fertility events were included in the study.

The data provided via the DDW application programming interface were loaded into the statistical program $\mathrm{R}$ version 3.2.5 (Ripley, 2001) and merged into one file. Male animals were excluded from the data set. Events were grouped by farm and cow identification number before being chronologically sorted. Events from the same cow that were recorded on different farms (due to selling and buying) were not merged.

\section{Deterministic Data Cleaning Method}

Triplet Creation. An alphabetical letter code (Table 1) was assigned to every fertility and mutation event to convert the time series data into a symbolic form (Aref et al., 2004). A single letter strand was configured for each cow by combining all letter codes (e.g., BHICH representing a birth, heat, insemination, calving, and heat event). The single letter strand was constructed in a chronological direction, from the oldest to the newest event (Figure 1). The letter strand was then divided into triplets. A triplet was defined as a letter sequence representing 3 consecutive events (Figure 1). By using 3 consecutive events instead of 2 , the relationship between the first and third event could be checked as well. For example, many cows show heat signs during pregnancy; accordingly, a farmer can record a heat event after a positive pregnancy check (PH; Sturman et al., 2000, Roelofs et al., 2010). The recording of a do not breed event after a heat event is also plausible. However, the sequence of events turns out to be suspicious when the 3 events are combined (PHD). Adding an extra event to the triplet sequence, creating a quadruple, would complicate interpretation as the number of relational combinations would increase 10 -fold.

The first triplet started at the oldest event and stopped when 2 consecutive events were added (e.g., $\mathrm{BHI}$ is the first triplet from the letter strand $\mathrm{BHICH}$ ). The next triplet within the same letter strand starts by moving up one place (e.g., HIC is the second triplet from the letter strand BHICH). This process was repeated until the last event of the letter strand has been used in a triplet (e.g., ICH being the last triplet from the letter strand $\mathrm{BHICH}$ ). An exception to the triplet definition was made if the total number of events was less than 3 . If only 1 or 2 events were recorded, then the letter code $\mathrm{X}$ (indicating a nonexisting event) was added to the letter strand until it was possible to cre- 


\begin{tabular}{llcccc} 
Event & Date & Letter code & Letter strand & $\begin{array}{c}\text { Triplet } \\
\text { number }\end{array}$ & $\begin{array}{l}\text { Triplet } \\
\text { code }\end{array}$ \\
\hline Birth & $08 / 29 / 2009$ & $\mathrm{~B}$ & 1 & $\mathrm{BHI}$ \\
Heat & $06 / 26 / 2010$ & $\mathrm{H}$ \\
Insemination & $11 / 11 / 2010$ & $\mathrm{I}$ & & $\mathrm{HIC}$ \\
Calving & $08 / 18 / 2011$ & $\mathrm{C}$ & & \\
Heat & $09 / 17 / 2011$ & $\mathrm{H}$ &
\end{tabular}

Figure 1. Shows the creation of a single letter strand by combining the letter codes of all the chronologically sorted mutation and fertility events from one single cow and the creation of triplets (a letter code of 3 consecutive events) from the letter strand. The initiation of the first triplet starts at the oldest event (left-most letter code) and stops when the 2 consecutive events are added to the oldest event. The start event for the next triplet is obtained by moving up one place in the letter strand. This process is repeated until the last event (right-most letter code) of the letter strand has been used in a triplet. Date: mo/d/yr.

ate at least one complete triplet (e.g., BXX or BHX). In total, 1,463 unique triplets could be constructed by combining all the different letter codes.

Labeling Triplets. Triplets were manually labeled by a domain expert as correct, suspicious, or impossible. All data quality rules applied to label the triplets are depicted in Table 2. The implementation of the data quality rules resulted in the construction of 131 correct, 390 suspicious, and 942 impossible triplets. A triplet was labeled as impossible if the sequence of events was physiologically impossible. The occurrence of a heat event before the birth is a clear example of such a triplet $(\mathrm{HBH})$. A suspicious triplet is a triplet where the sequence of events is physiologically possible; however, one or more events seem to be missing, for

Table 1. The alphabetical letter code assigned to the mutation and reproduction events

\begin{tabular}{lll}
\hline Event & $\begin{array}{l}\text { Alternative } \\
\text { naming }^{1}\end{array}$ & Letter code \\
\hline Mutation event & & B \\
Birth & Obtained & O \\
Bought & Mortality & S \\
Sold & M \\
Death & & H \\
Reproduction event & & I \\
Heat & & A \\
Insemination & \\
Abortion & & P \\
Positive pregnancy check & & N \\
Negative pregnancy check & & D \\
Calving & & D \\
Do not breed & &
\end{tabular}

${ }^{1}$ An alternative naming had to be provided to prevent the duplication of a letter code.

${ }^{2}$ Natural mating, AI, and embryo transfer were all classified as an insemination event. example a triplet constructed of 3 consecutive calving events (CCC). It is physiologically possible for a cow to deliver 3 times; however, inseminations appear to be missing because a cow needs at least one insemination to become pregnant. If the sequence of 3 events was physiologically possible and no data were clearly missing, then the triplet was labeled as correct. If the first triplet did not contain a birth event (B), then it was labeled as impossible. A triplet error rate was calculated as the sum of all suspicious and impossible triplets divided by the total number of triplets.

\section{Probabilistic Data Cleaning Method}

Correct or Incorrect Reproductive Record. Cows were labeled in a categorical manner, as having either correct or incorrect reproductive data. An animal labeled as correct had only correct triplets, whereas an animal was labeled as incorrect if it had at least one suspicious or impossible triplet. The binary correct/ incorrect variable was used as the response variable to be predicted.

Predictor Variable Selection. For each cow a set of 16 predictor variables was collected. All variables were used for the prediction of the binary response variable. The variables are listed in Table 3, containing a definition of the variable, the data type of the variable, and the level of collection (cow or farm level). Cow identification number, the binary response variable and the predictor variables were merged into a data set with 18 dimensions. The data set was split into a training $(60 \%)$ and test set (40\%) using the "sample" function from the package "base" for R (Ripley, 2001).

Random Forests and Penalized Logistic Regression Model. The RF algorithm (Breiman, 2001) 
Table 2. Rules applied to classify the triplets

\begin{tabular}{lll}
\hline Event & Rule applied & Result \\
\hline Mutation event & No multiple events & Impossible \\
Birth (B) & No event before a birth event & Impossible \\
& No multiple events & Impossible \\
Death & No event after a death event & Impossible \\
& No multiple events without a sold event & Impossible \\
Bought (O) & No multiple events without a bought event & Impossible \\
Sold (S) & Only event after a sold event is a bought event & Impossible \\
& & \\
Reproduction event & No consecutive events & Suspicious \\
Heat & After a positive pregnancy check or an insemination & Suspicious \\
Abortion & After an insemination & Suspicious \\
& After an insemination followed by a heat event & Suspicious \\
Positive pregnancy check (P) & After a positive pregnancy check & Suspicious \\
& After an insemination & Suspicious \\
Negative pregnancy check & After an insemination followed by a heat event & Suspicious \\
& After a negative pregnancy check & Suspicious \\
Auspicious & Suspicious \\
Calving (C) & After an abortion & Suspicious \\
Do not breed & After a positive pregnancy check & Suspicious \\
Suspicious & Impossible \\
Insemination (I) & After a nositive pregnancy check followed by a bought event & \\
\hline
\end{tabular}

${ }^{1}$ Only applicable within one triplet (BOSO), not for BOIIPCO.

implemented in the "randomForest" package (Liaw and Wiener, 2002) within the R environment, was used to predict the binary response variable (correct or incorrect reproduction data). The $\mathrm{RF}$ is a classification and regression algorithm that groups multiple individual decision trees. Each of the individual trees in the RF is constructed by using bootstrap aggregation (bagging) and random variable selection (Breiman, 2001; Strobl et al., 2007). Tree tuning parameters have to be specified for the RF algorithm (Liaw and Wiener, 2002). In our study, the random seed was set on 123 and the classification function was selected as the RF type.

Table 3. The 16 variables used for the prediction of the binary response value

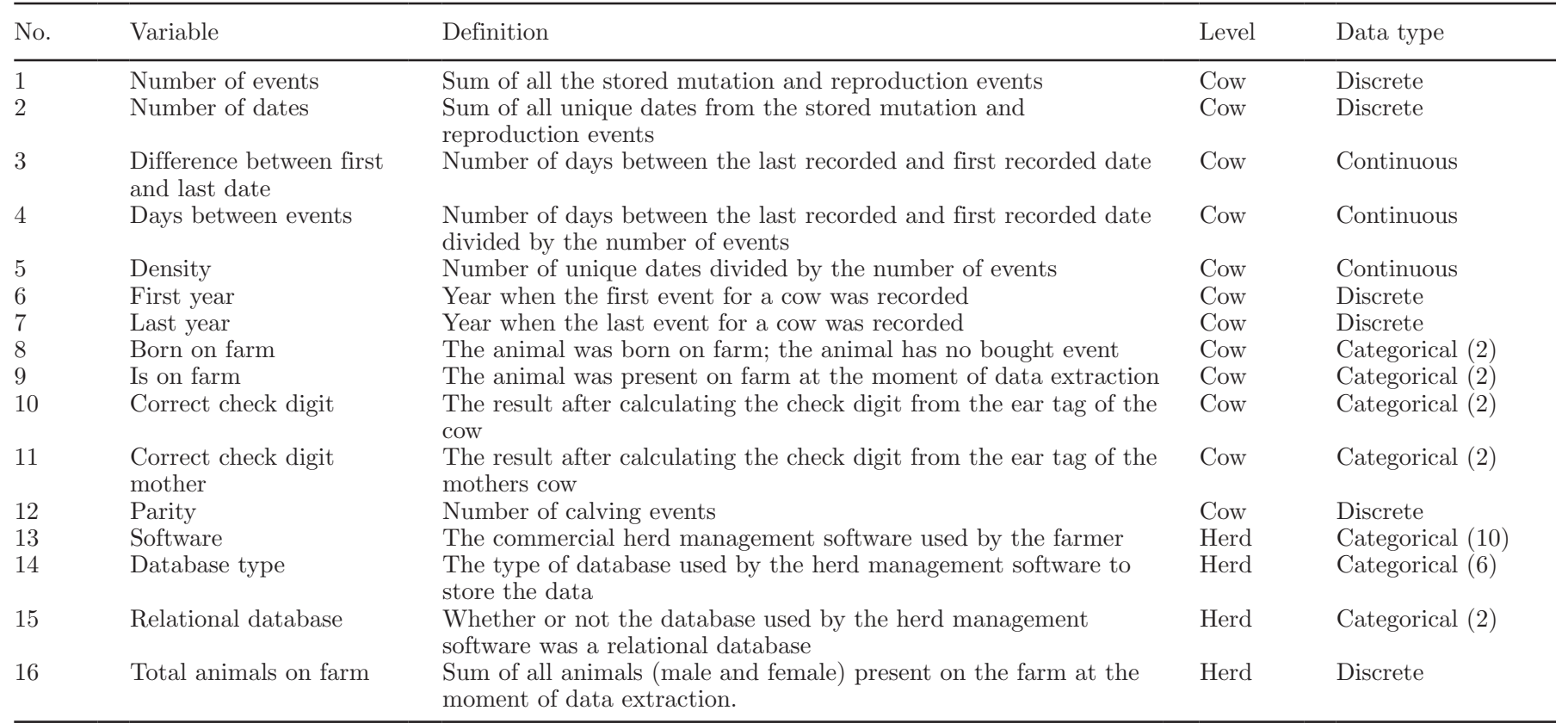


Sampling was done with replacement. The number of variables randomly sampled as candidates at each split (mtry) was set as the square root of the number of variables in the data set. The number of trees to be grown (ntree) was set at 2,000. The minimum size of the end nodes (nodesize) was set on 1 , the default value for classification (Liaw and Wiener, 2002).

The "penalized" package (Goeman et al., 2010) for R was used to create a PLR. A naïve elastic net penalty was calculated for the PLR. The naïve elastic net is a combination of a lambda1 absolute value ("lasso") penalty (Tibshirani, 1996) and a lambda2 quadratic ("ridge") penalty (Hoerl and Kennard, 1970; Le Cessie and Van Houwelingen, 1992; Verweij and Van Houwelingen, 1994). The optimal value for lambda1 and lambda2 was obtained via the "optL1" and "optL2" function implemented in the penalized package. The training set and all 16 predictor variables were used for building the RF and the PLR.

\section{Evaluation Methods}

The test set was used for the evaluation of the predictive performance of the RF and the PLR toward the binary response variable (correct or incorrect reproduction data). For each case of the test set, the response value or class was predicted by running it down the RF and PLR model. The predicted class of each case was compared against the true class. Results from this comparison were put into a confusion or error matrix. The predicted classes for the test samples were used to calculate the 4 categories of the confusion matrix: the true positives (TP), the false positives (FP), the true negatives (TN), and the false negatives (FN; Fawcett, 2006).

A receiver operating characteristic (ROC) curve for both models was constructed via the package "ROCR" (Sing et al., 2005). For the 2-dimensional ROC curve, the true positive rate (TPR) was plotted on the $\mathrm{y}$ axis and the false positive rate (FPR) was plotted on the $\mathrm{x}$-axis (Fawcett, 2006). The TPR represented the fraction of positive examples that are correctly predicted as positive $[\mathrm{TPR}=\mathrm{TP} /(\mathrm{TP}+\mathrm{FN})]$. The FPR represented the fraction of negative cases that are incorrectly predicted as positive $[\mathrm{FPR}=\mathrm{FP} /(\mathrm{FP}$ $+\mathrm{TN})$ ]. Additionally, the associated area under the receiver operating characteristic curve was transformed into a single scalar value (AUC; Bradley, 1997). Two performance measurements were calculated: the total prediction accuracy (ACC) and the precision or positive predictive value (PPV). The ACC measures the predictive correctness $[=(\mathrm{TP}+\mathrm{TN}) /(\mathrm{TP}+\mathrm{TN}+\mathrm{FP}$ $+\mathrm{FN})]$. The PPV reflects the percentage of animals that are correctly predicted as having correct data $[=$ $\mathrm{TP} /(\mathrm{TP}+\mathrm{FP})]$.

The "randomForest" package (Liaw and Wiener, 2002) is able to calculate measures for variable importance. In our study, the "permutation importance measure" or "mean decrease in accuracy" was used to compute the influence of the predictor variables on the RF. The mean decrease in accuracy was calculated as the difference in prediction accuracy before and after permuting the prediction variable $\mathrm{X}_{\mathrm{j}}$, averaged over all trees (Breiman, 2001).

The influence of the individual variable on the classification algorithm was plotted using the "partialPlot" function from the "randomForest" package (Liaw and Wiener, 2002). The partial dependence for the 6 predictor variables with the highest mean decrease in accuracy was examined. Partial dependence visualizes the dependency of the probability that a cow was classified as having correct reproduction data, based on a single predictor variable after averaging the effects of all other predictors in the model.

\section{RESULTS}

\section{Descriptive Data}

Data of 39,227 animals were obtained from 26 different farms. The average number of animals on farm (male and female animals) at the time of data extraction was $240(\mathrm{SD}=148)$. The largest farm had 688 animals and the smallest farm had 101 animals. Fortyeight percent of all animals were excluded (stored as male animals), leaving 20,280 female animals (52\%) for further research. The date from the gathered events ranged from March 1972 to March 2016. Nine out of 26 farms $(35 \%)$ started with data collection after January 1,2000 . Thirty-one percent or 8 out of the 26 farms initiated data collection in the 1990s. Only 187 of all female animals had events recorded before January 1 , 1980. The nulliparous animals represented $44 \%$ of all female animals in our data set. The distribution of animals per parity was 3,717 for parity $1,2,741$ for parity $2,2,000$ for parity 3 , and 2,857 cows for a parity higher than 3 . The average parity in the data set was 1.5 (SD $=1.8$ ).

\section{Deterministic Data Cleaning Method}

In total, 150,443 triplets were created. The median number of triplets constructed per cow was 6 , corresponding to the recording of 8 mutation or fertility events (or both) per cow. The minimum number of triplets per cow was 1 . The maximum number of 


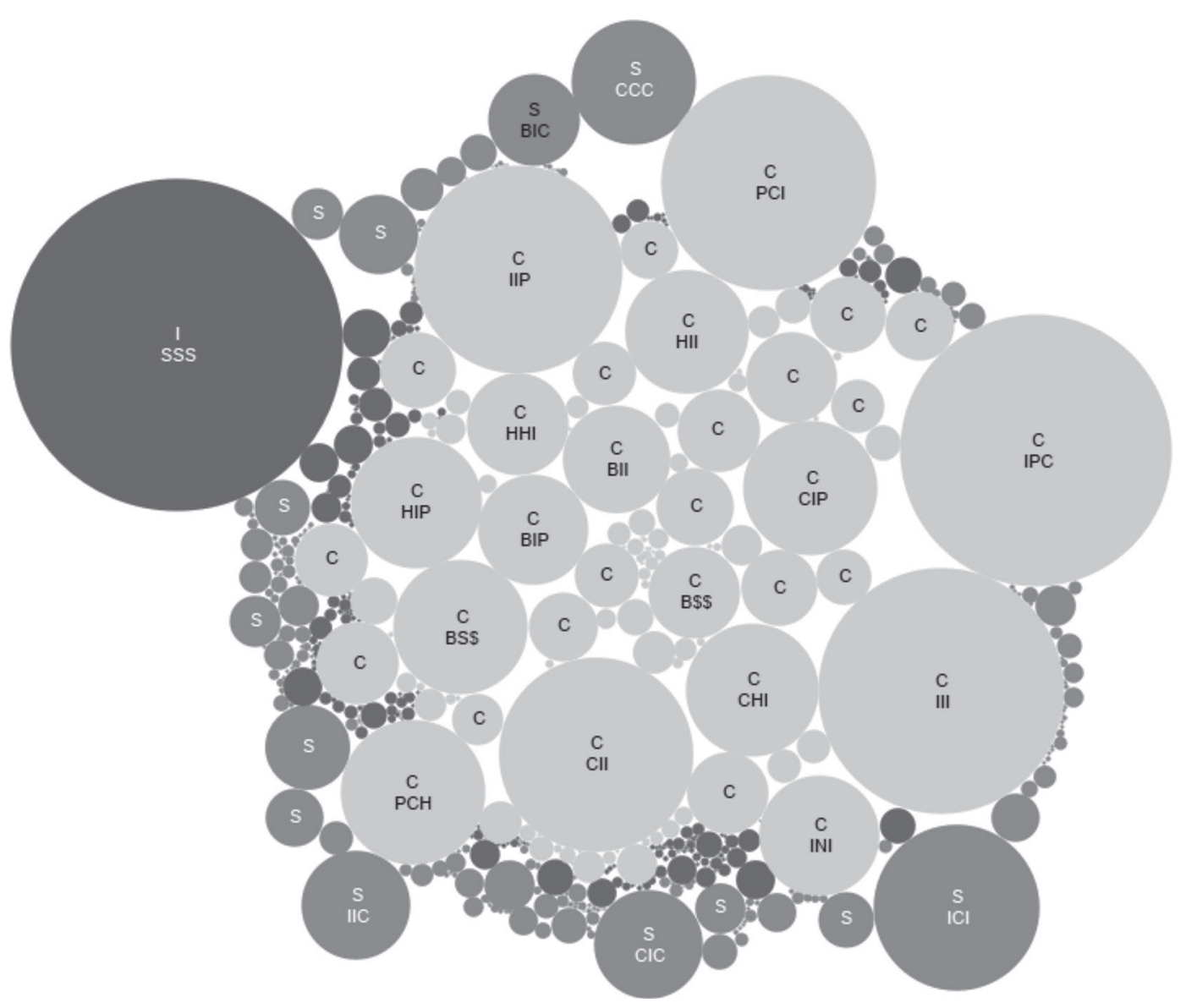

Figure 2. A packed bubble graph visualizing the distribution of the 539 unique triplets according to their labeling: correct (light gray), suspicious (intermediate gray), and incorrect (dark gray). The size of the bubble refers to the number of times the triplet was created in the study. See Table 1 for definitions of letter codes; $\$$ represents the letter code X (a non-event).

triplets per cow was 346. Less than $0.51 \%$ (104 out of 20,280 ) of all female animals had several triplets higher than 100. Ninety-two percent of all cases with a triplet number higher than 100 belonged to the same farm. From the 150,443 triplets, $65.4 \%$ were labeled as correct, $17.4 \%$ as suspicious, and $17.2 \%$ as impossible, which leaded to a triplet error rate of $35 \%$. Altogether, $37 \%$ of all the possible triplets (539 out of 1,463$)$ were found. From the 539 unique triplets, $44 \%$ were labeled as suspicious, $37 \%$ as impossible, and $19 \%$ as correct. The distribution of the detected triplets is shown in Figure 2 (a clarification for the alphabetical letter code can be found in Table 1). The most reported triplet was SSS (10.7\%), the second most reported triplet was IPC $(8.5 \%)$, and the third most reported triplet was III (7.1\%). The letter code X (nonevent) was used at least one time in 7,344 triplets or in $5 \%$ of all triplets. Birth was the first event in 5,936 (81\%) of the X containing triplets. Calving and obtained were the first event in, respectively, $706(10 \%)$ and $443(6 \%)$ of the triplets with a nonevent. For 3,758 or $18 \%$ of the animals, the first triplet did not contain a birth event. In $55 \%$ of these first triplets, an obtained $(\mathrm{O})$ event was enclosed in the triplet. Out of the 3,758 first triplets without a birth event, 3,479 were immediately labeled as a suspicious or impossible triplet.

\section{Probabilistic Data Cleaning Method}

According to our definition, $52 \%$ of the cows were labeled as having incorrect reproduction data and $48 \%$ of the cows were labeled as having correct reproduction data. After subsampling the overall data set, the training set contained 14,196 animals and the test set contained 6,084 animals. For both the test and training set, the distribution of correct and incorrect animals was 48 versus $52 \%$.

Figure 3 compares the ROC curves from the RF and the PLR. The AUC value based on the ROC curve was 0.969 for RF and 0.81 for PLR. The RF had a higher ACC in comparison with the PLR (89 vs. $72 \%$ ). The PPV for RF was 90 and $75 \%$ for PLR. 


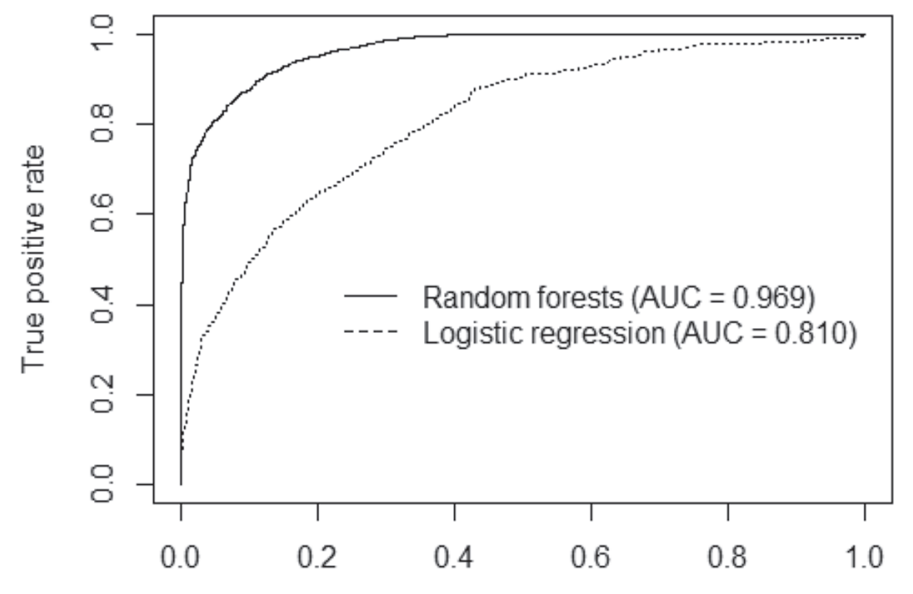

False positive rate

Figure 3. The receiver operating characteristic and the area under the curve (AUC) values based on the prediction performance of the random forests algorithm and penalized logistic regression. The y-axis represented the fraction of positive examples that are correctly labeled as positive $[\mathrm{TPR}=\mathrm{TP} /(\mathrm{TP}+\mathrm{FN})]$. The $\mathrm{x}$-axis represented the fraction of negative cases that are misclassified as positive $[\mathrm{FPR}=\mathrm{FP}$ $(\mathrm{FP}+\mathrm{TN})] . \mathrm{TPR}=$ true positive rate; $\mathrm{TP}=$ true positive; $\mathrm{FN}=$ false negative; $\mathrm{FPR}=$ false positive rate; $\mathrm{FP}=$ false positive; $\mathrm{TN}=$ true negative.
A ranked list of all 16 variables used for prediction and their influence on the accuracy of the RF model is shown in Figure 4. The 2 most influential variables are born on farm and farm size. The third most influential variable is days between events, having a comparable influence as parity.

Figure 5 depicts the partial dependence on the class "correct reproduction data" for the predictor variables: born on farm, farm size, parity, days between events, first year, and density. Being born on farm has a positive effect on having correct data. From the 17,057 animals being born on farm, $55 \%$ had correct fertility data. Only $9 \%$ of the animals purchased from another farm had correct fertility data. An animal had the highest probability of being classified as having correct fertility data if the farm size ranged between 250 and 400 animals. Nulliparous animals had the highest probability of having only correct triplets. The recording of a calving event has a negative effect on the quality of reproduction and mutation data (Figure 5). The percentage of animals with correct data dropped from $78 \%$ for nulliparous animals to $32 \%$ for primiparous animals. Every additional calving caused an extra $5 \%$ decline in the percentage of animals with correct data. An aver-

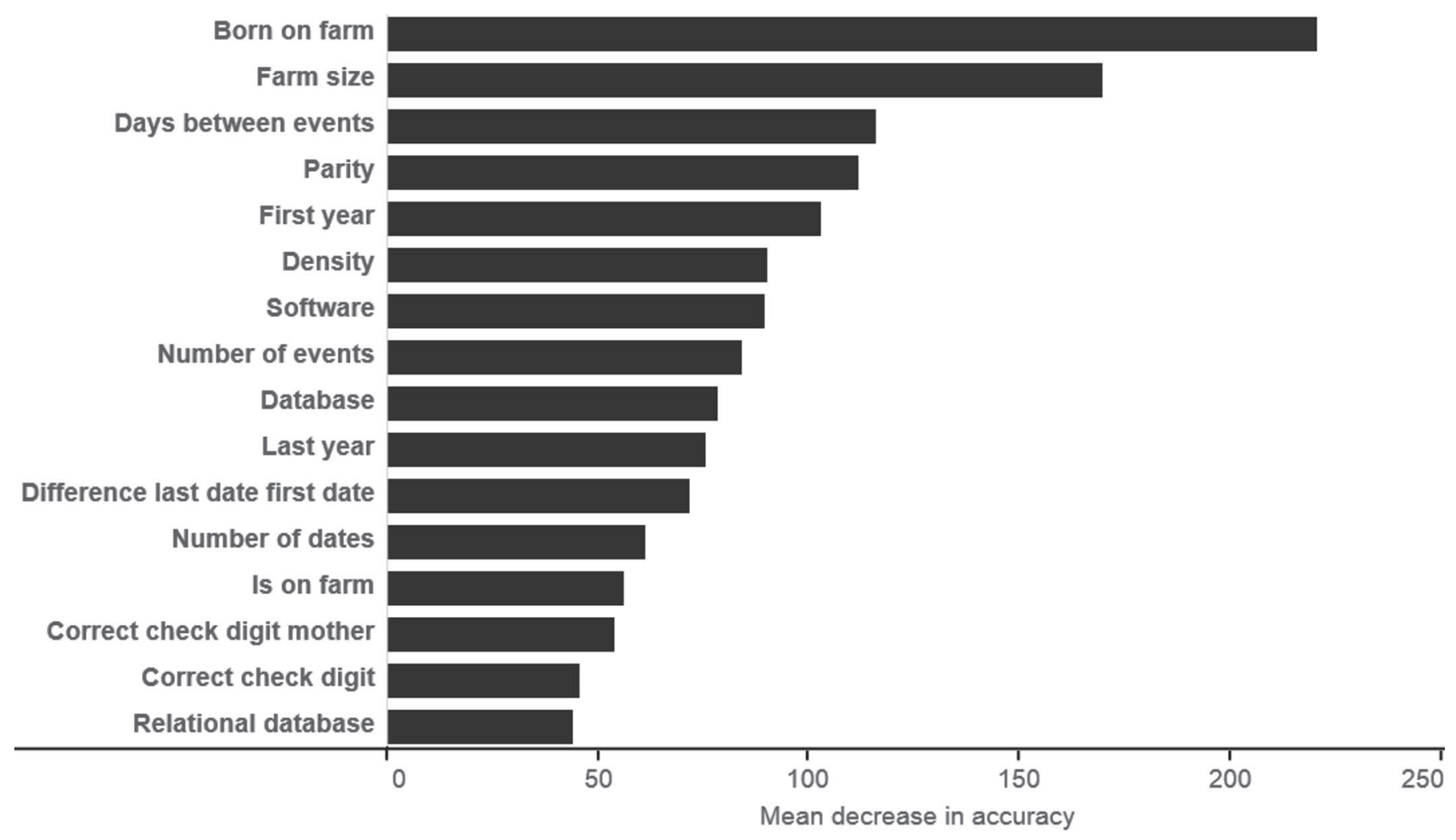

Figure 4. A ranked list of the 16 variables used for prediction and their influence on the accuracy of the random forests model. The mean decrease in accuracy was calculated as the difference in prediction accuracy before and after permuting the prediction variable $\mathrm{X}_{\mathrm{j}}$, averaged over all trees. 
Partial dependency plots for the class correct reproduction data
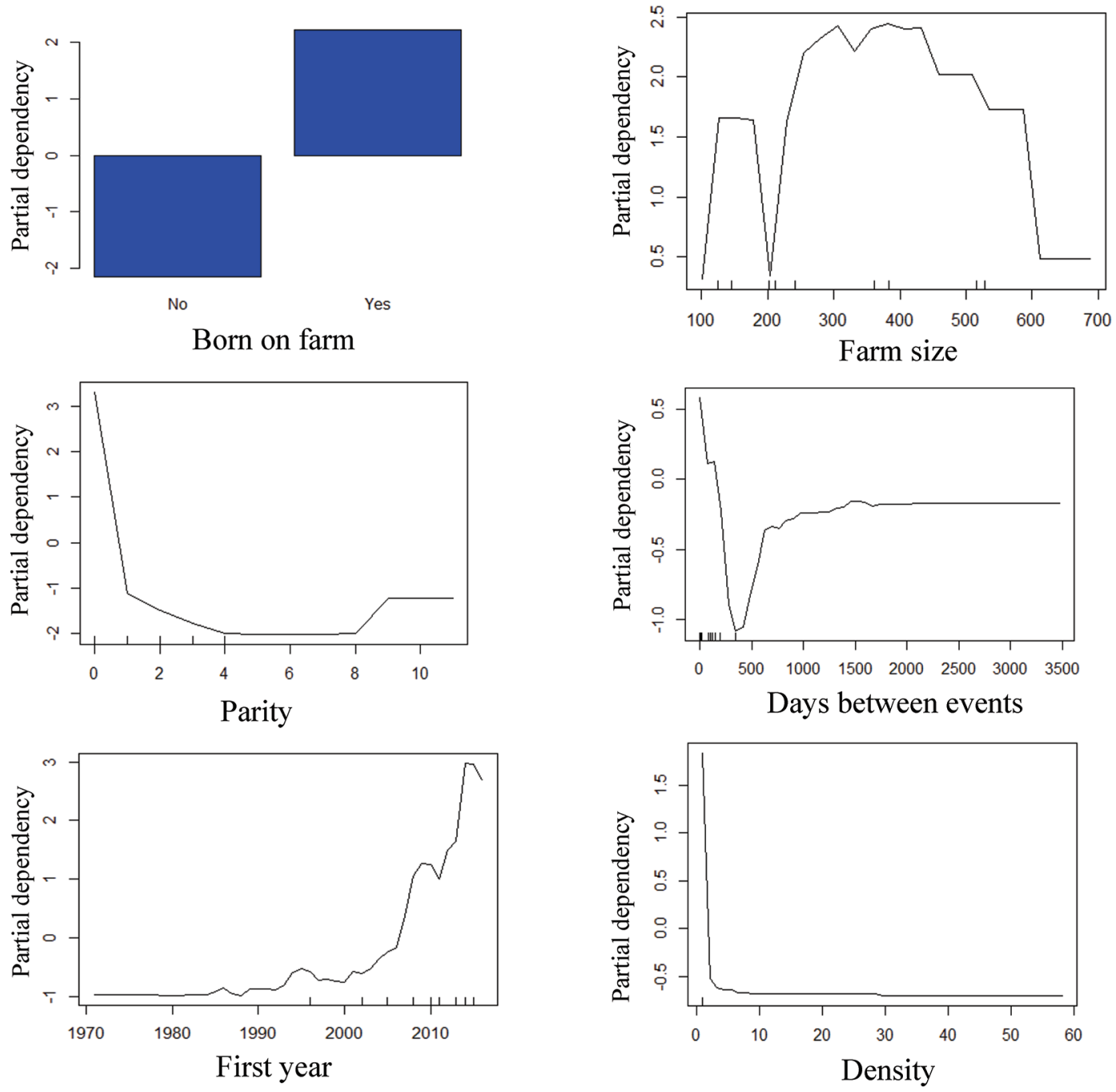

Figure 5. Partial dependence plots for the 6 predictor variables with the highest mean decrease in accuracy. The x-axis represents the predictor variables, and the y-axis represents the partial dependence. Partial dependence visualizes the dependency of the probability that a cow was classified as having correct reproduction data, based on a single predictor variable after averaging the effects of all other predictors in the model. Color version available online.

age of more than $180 \mathrm{~d}$ between the recorded events has a distinct negative effect on the correctness of the data (Figure 5). The biggest negative effect occurred when the time between events ranged around $410 \mathrm{~d}$. The probability of being classified as having correct fertility data was higher if the first event of a cow was recorded after 2008. Out of all the animals with correct data, $86 \%$ had their first event recorded after January 1,2006 . The percentage of animals with correct data declined with $1 \%$, for every year the first recorded event aged. The decline was much more pronounced, $7 \%$ per year, for the 10-yr period from 2016-2006. Animals had a higher probability of having impossible or suspicious triplets if more than one event was recorded on the same date (a density $>1$ ). Seven out of 10 animals $(1,420$ out of 1,992) animals with a density higher than 1 had incorrect data.

\section{DISCUSSION}

\section{Data Quality}

A generally accepted approach for defining data quality is the concept of fitness for use, emphasizing the data consumers' perspective, whether or not the data can be used (Tayi and Ballou, 1998). The fitness for use statement causes data quality to be a nebulous term (Wand and Wang, 1996). Each data consumer will 
have individual expectations and standards. Reconciling each and every expectation or standard into an overall data quality definition will hardly be executable (Orr, 1998). We suggest that a correct physiological occurrence of reproductive events should be the minimum data quality standard.

The consequences for the reliability, credibility, and integrity of the data differ depending on the data error. An error with regard to a mutation event will always lead to an impossible sequence of events. On the contrary, an error toward a fertility event will often cause a suspicious sequence of events (Table 2). For example, the nonrecording of an insemination between 2 consecutive calving events (PCC) only influences the credibility and integrity of the last calving event. On the other hand, the recording of a calving event before the day of birth (CBI) will compromise all events recorded for that animal.

\section{Deterministic Data Cleaning Method}

Triplets. By using triplets, we focused on the completeness and correctness of the sequence from 3 consecutive events. Completeness and correctness are 2 commonly used data quality dimensions in dairy research (Green and Kao, 2007; Mörk et al., 2010; Espetvedt et al., 2012; Espetvedt et al., 2013).

The completeness dimension assesses whether a data collection has values present for all attributes that are expected to have values (Kim et al., 2003; Li et al., 2014). Our aim was to spot a physiologically missing event or a sequence of events that suggests that an event is missing. A nice example for a clearly missing event is the recording of a calving event without the recording of an insemination event $(\mathrm{CCH})$. We focused on the presence of a birth event in the first triplet. The reason for this is that if no birth event was recorded and all triplets of a cow were labeled as correct, the cow would be mistakenly labeled as correct. The absence of a birth event in the first triplet was penalized, preventing the occurrence of a FP label. Only $7 \%$ of the animals without a birth event in the first triplet had a physiologically correct first triplet, indicating a high probability that, if the birth event is missing in the first triplet, the sequence of the first 3 events will be incorrect.

The correctness dimension deals with satisfying all the constraints of a data value for an attribute or entity (Kim et al., 2003; Li et al., 2014). We focused on the relational constraints that arise when fertility and mutation events are put into relation with each other. The recording of a heat event before the day of birth $(\mathrm{HBH})$ or the recording of an abortion event after the animal has died (CMA) is undoubtedly not possible.
Almost $20 \%$ of all triplets had a physiologically impossible sequence of events. Hence, it is plausible to assume that a very small number of data quality rules are implemented in dairy herd management software. The presence of a physiologically impossible sequence of events forces the data user to be trapped between disregarding only one animal or not trusting the data from all animals.

The triplet method does validate the accuracy of an event. The absence of a real connection between the end user and the data capturing process creates a setting where the data user has no control on the accuracy of this process (Sorensen et al., 1996). Hence, it is hardly possible to verify if a mutation or fertility event stored in a secondary database actually occurred on a specific date.

Triple Count and Visualization. The most common correct triplets depicted in Figure 2 (IPC, III, IIP, $\mathrm{CII}, \mathrm{PCH}$ ) suggest an insemination, a positive pregnancy check, a heat, and a calving event to be the most important fertility events. This could be explained by a higher physiological occurrence of these events.

A small percentage of animals had an extremely high number of triplets. Almost all of these animals belonged to the same farm and had multiple, up to 100, consecutive sold events. As such, approximately 100 animals were responsible for the high number of times the impossible triplet SSS was detected. We have reason to believe that the SSS error is either caused by incorrect usage of the software or by a default in the software code.

Only $37 \%$ of all possible triplets were detected; this does not connote the nonexistence of the remaining triplets. It only suggests that the combination of events in these triplets are rare. A triplet error rate of $35 \%$ is at the higher end. In literature, (field) error rates in different industries range from $0.5 \%$ up to $30 \%$.

Fertility Data Collection Rating. The triplet analysis could be used to accredit a data quality rating analogous to the (milk production) data collection rating system used by the Council on Dairy Cattle Breeding in the United States. One could rate an individual animal by calculating the rate of correct triplets (number of correct triplets divided by the number of all triplets generated for the animal). Rating the farm could be done by calculating the rate of correct animals (= number of correct animals divided by all animals) or by calculating the rate of impossible and suspicious triplets (sum of all impossible and suspicions triplets divided by all correct triplets). As such, herds could be classified into different data quality classes (poor, medium, high, excellent) or rank them according to their data quality rate. Hence, potential users can decide to only extract data from herds with a minimum 
data quality standard (class high or excellent). Farmers could be offered a higher data collection payment if they are ranked within the top 100 farms or depending on the data quality class to which they have been assigned.

\section{Probabilistic Data Cleaning Method: Correct or Incorrect Reproductive Record}

The definition of a correct animal as having $100 \%$ biologically sensible triplets throughout her lifetime is very strict and could introduce selection bias as older animals are more likely to have incorrect data. A plausible explanation is the length of the opportunity period in which the farmer has a chance to make a data recording error. Typically, a yearling heifer has no more than 3 or 4 events, so probability for errors are minimal. A 7-yr-old cow has dozens of events, increasing the probability that the farmer will make at least one error or omission somewhere during her lifetime.

We suggest remaining very reticent when using incorrect reproductive records for research purposes. Allowing data errors or omitting incorrect subsequences will have consequences toward the reliability of the data and most importantly could influence the outcome of calculations. Comparing whether the result of a calculation will significantly differ if only $100 \%$ correct records are used or when data errors are allowed could solve the latter. Data errors are easily identified by using the triplet method, hence one can quickly include or exclude animals with specific data errors. At this end, it is up to the data user to assess whether only correct animals can be used or a less strict inclusion can be applied. According to our definition, $52 \%$ of the cows were labeled as having incorrect reproduction data. Comparable rates (50 to $80 \%$ ) of inaccurate, incomplete, or ambiguous computerized records in other industries are mentioned by Strong et al. (1997).

\section{Random Forest Versus Penalized Logistic Regression}

The second objective of the present study was to develop a probabilistic data cleaning system based on cow- and farm-related variables. To perform the deterministic triplet method, multiple data manipulations have to be performed. All data manipulation will be in vain if after data cleaning the data appear to be incorrect. By only assessing data with a high potential of being correct, the time and costs spent on data cleaning can be reduced considerably (Haug et al., 2011).

The logistic regression was penalized to prevent overfitting due to collinearity of the variables or due to high-dimensionality of the data set (Goeman et al.,
2010). The probability that correlated prediction variables are used together is minimized as the RF samples from the potential explanatory variables rather than fitting them all simultaneously (Breiman, 2001). As such, overfitting will only occur in a limited amount of trees and the effect of overfitting will be averaged over all the trees of the RF.

The predictive properties of a classical prediction model (PLR) was compared with a popular and widely used machine learning algorithm (RF). The prediction capabilities of machine learning algorithms are known to exceed classical statistical methods (Shahinfar et al., 2014), especially when complex relations within a data set occur (Strobl et al., 2009). The better predictive performance of the $\mathrm{RF}$ is confirmed by a superior ROC curve, a higher AUC, a better ACC, and a higher PPV in comparison with the PLR method. The inferior predictive performance of the PLR could be explained by the absence of a linear relationship in some predictor variables, for example, farm size and days between events (Figure 5).

Implementation of the RF could preselect animals (early stage data cleaning), as fewer animals with incorrect data would be presented for actual deterministic data cleaning. The time and cost for data cleaning would decline because 9 out of 10 animals selected via the RF would actually have correct data.

Predictor variables were chosen so they could be extracted directly from the data set and no additional variable collection had to be done. Often secondary data are anonymized (Safran et al., 2007) or it is impractical to collect additional information (Hox and Boeije, 2005). The farm identification number as such was not used as a predictor variable. Hence the RF method can be generalized and used to assess data from other farms as well.

Using fertility data from animals that are not born on farm should be discouraged. Ninety percent of the animals that are not born on farm have incorrect fertility data, suggesting that farmers do not record data retrospectively. If an animal is purchased, the farmer will only start collecting data from the moment the animal has been obtained.

The implemented management could be a possible explanation why farm size has a significant influence on the correctness of the data. We were unable to test this hypothesis, as we did not gather any information about the data capturing process. Farms with more than 250 animals might have staff responsible for recording data and the capturing process might be more standardized (Leahy et al., 2004). Animals housed in farms with a size ranging around 200 animals have a higher probability of having missing or incorrect data. A plausible explanation is that a farm with 200 animals is still 
managed by one person, creating a high workload. The high workload might cause more erroneous data capturing (Glaser et al., 1999; Parker et al., 2013). The first parturition causes the biggest decline in percentage of animals with correct data. Every additional calving has the same negative effect on the quality of the data. A possible explanation why the first parturition has such an effect on data quality is the low number of events recorded before the first calving event (Sartori et al., 2010). In our study, $67 \%$ of all nulliparous animals had only 1 or 2 events recorded. As a consequence, only $11 \%$ of all possible triplets $(156$ out of 1,463$)$ could be applied to check the correctness of the letter sequence. As soon as a calving event or a third event is recorded, all 1,463 different triplets can be constructed. Hence, the number of implemented data quality rules increased 10 -fold. One could consider waiting until the first calving event is recorded before using fertility data for research about youngstock reproduction. However, this would exclude unfertile youngstock (no calving event) for research purposes. Another possibility could be to wait 36 mo after the day of birth to evaluate fertility events of youngstock.

We suggest to be careful when using data from more than $10 \mathrm{yr}$ in the past. Only $14 \%$ of the animals with correct data had a first event recorded more than 10 yr ago. The overall quality of the data can rapidly decrease if the quality of data input and recording is not controlled (Batini et al., 2009). The error rate of a data set will increase every year by $5 \%$ if no actions are undertaken to preserve the quality of the data. A higher increase in error rate $(7 \%)$ was detected compared with the literature, for the time period 2016-2006. A possible explanation could be that dairy herd management software is developed mainly for management purposes and not specifically for data storage.

\section{CONCLUSIONS}

A correct and physiological occurrence of events should be the minimum quality standard for fertility data available on a dairy herd. The triple method was shown to be a good way to reduce the dimensionality of reproduction data in an easily understandable manner. The RF was demonstrated to be a good and fast method for predicting the correctness of the fertility data stored in dairy herd management software. More than $50 \%$ of the records stored in dairy herd management software have systematic or random errors (or both). The quality of fertility data stored in dairy herd management software should be reviewed before being used for research purposes. One should be cautious when using fertility data from dairy herd management software if an animal is not born on the farm, an animal is older than $10 \mathrm{yr}$, or the animal is nulliparous.

\section{REFERENCES}

Aref, W. G., M. G. Elfeky, and A. K. Elmagarmid. 2004. Incremental, online, and merge mining of partial periodic patterns in time-series databases. IEEE Trans. Knowl. Data Eng. 16:332-342.

Arts, D. G., N. F. De Keizer, and G.-J. Scheffer. 2002. Defining and improving data quality in medical registries: A literature review, case study, and generic framework. J. Am. Med. Inform. Assoc. 9:600-611.

Ballou, D. P., and H. L. Pazer. 1985. Modeling data and process quality in multi-input, multi-output information systems. Manage. Sci. $31: 150-162$.

Batini, C., C. Cappiello, C. Francalanci, and A. Maurino. 2009. Methodologies for data quality assessment and improvement. ACM Comput. Surv. 41:3-16.

Bradley, A. P. 1997. The use of the area under the ROC curve in the evaluation of machine learning algorithms. Pattern Recognit. 30:1145-1159.

Breiman, L. 2001. Random forests. Mach. Learn. 45:5-32.

Caraviello, D., K. Weigel, M. Craven, D. Gianola, N. Cook, K. Nordlund, P. Fricke, and M. Wiltbank. 2006. Analysis of reproductive performance of lactating cows on large dairy farms using machine learning algorithms. J. Dairy Sci. 89:4703-4722.

Dairy Data Warehouse. 2016. Accessed Jul. 1, 2016. https://www. dairydatawarehouse.com/index.html.

Espetvedt, M., O. Reksen, S. Rintakoski, and O. Østerås. 2013. Data quality in the Norwegian dairy herd recording system: Agreement between the national database and disease recording on farm. J. Dairy Sci. 96:2271-2282.

Espetvedt, M., C. Wolff, S. Rintakoski, A. Lind, and O. Østerås. 2012. Completeness of metabolic disease recordings in Nordic national databases for dairy cows. Prev. Vet. Med. 105:25-37.

Fawcett, T. 2006. An introduction to ROC analysis. Pattern Recognit. Lett. 27:861-874.

Fourichon, C., H. Seegers, and X. Malher. 2000. Effect of disease on reproduction in the dairy cow: A meta-analysis. Theriogenology 53:1729-1759.

Glaser, D. N., B. C. Tatum, D. M. Nebeker, R. C. Sorenson, and J. R. Aiello. 1999. Workload and social support: Effects on performance and stress. Hum. Perform. 12:155-176.

Goeman, J., R. Meijer, and N. Chaturvedi. 2010. L1 penalized estimation in the Cox proportional hazards model. Biom. J. 52:70-84.

Green, D. M., and R. Kao. 2007. Data quality of the Cattle Tracing System in Great Britain. Vet. Rec. 161:439-443.

Harpe, S. E. 2009. Using secondary data sources for pharmaco-epidemiology and outcomes research. Pharmacotherapy 29:138-153.

Haug, A., F. Zachariassen, and D. Van Liempd. 2011. The costs of poor data quality. J. Ind. Eng. Manage. 4:168-193.

Hoerl, A. E., and R. W. Kennard. 1970. Ridge regression: Biased estimation for nonorthogonal problems. Technometrics 12:55-67.

Hox, J. J., and H. R. Boeije. 2005. Data Collection, Primary Versus Secondary. Pages 593-599 in Encyclopedia of Social Measurement 1st ed. Academic Press, San Diego, CA.

Kim, W., B.-J. Choi, E.-K. Hong, S.-K. Kim, and D. Lee. 2003. A taxonomy of dirty data. Data Min. Knowl. Discov. 7:81-99.

Le Cessie, S., and J. C. Van Houwelingen. 1992. Ridge estimators in logistic regression. J. R. Stat. Soc. Ser. C Appl. Stat. 41:191-201.

Leahy, H., D. J. Ruane, and E. G. O'Riordan. 2004. An investigation into the impact of farm labour use on Irish suckler beef farms. Pages 519-530 in Proc. 20th Annual AIAEE Conference, Dublin, Ireland.

Li, L., T. Peng, and J. Kennedy. 2014. A rule based taxonomy of dirty data. GSTF Int. J. Comput. 1:140-148.

Liaw, A., and M. Wiener. 2002. Classification and regression by randomForest. R News 2:18-22. 
Lim, T.-S., W.-Y. Loh, and Y.-S. Shih. 2000. A comparison of prediction accuracy, complexity, and training time of thirty-three old and new classification algorithms. Mach. Learn. 40:203-228.

Loeffler, S. H., M. J. de Vries, and Y. N. Schukken. 1999. The effects of time of disease occurrence, milk yield, and body condition on fertility of dairy cows. J. Dairy Sci. 82:2589-2604.

Loh, W. Y. 2011. Classification and regression trees. Wiley Interdiscip. Rev. Data Min. Knowl. Discov. 1:14-23.

López-Gatius, F., P. Santolaria, I. Mundet, and J. L. Yaniz. 2005. Walking activity at estrus and subsequent fertility in dairy cows. Theriogenology 63:1419-1429.

Mörk, M. J., C. Wolff, A. Lindberg, I. Vågsholm, and A. Egenvall. 2010. Validation of a national disease recording system for dairy cattle against veterinary practice records. Prev. Vet. Med. 93:183192.

Orr, K. 1998. Data quality and systems theory. Commun. ACM 41:6671.

Parker, S. L., N. L. Jimmieson, and C. E. Amiot. 2013. Self-determination, control, and reactions to changes in workload: A work simulation. J. Occup. Health Psychol. 18:173-190.

Pipino, L. L., Y. W. Lee, and R. Y. Wang. 2002. Data quality assessment. Commun. ACM 45:211-218.

Rahm, E., and H. H. Do. 2000. Data cleaning: Problems and current approaches. IEEE Data Eng. Bull. 23:3-13.

Ripley, B. D. 2001. The R project in statistical computing. MSOR Connections. The Newsletter of the LTSN Maths, Stats \& OR Network 1:23-25.

Roelofs, J., F. López-Gatius, R. Hunter, F. Van Eerdenburg, and C. Hanzen. 2010. When is a cow in estrus? Clinical and practical aspects. Theriogenology 74:327-344.

Safran, C., M. Bloomrosen, W. E. Hammond, S. Labkoff, S. MarkelFox, P. C. Tang, and D. E. Detmer. 2007. Toward a national framework for the secondary use of health data: An American Medical Informatics Association White Paper. J. Am. Med. Inform. Assoc. 14:1-9.

Sartori, R., M. R. Bastos, and M. C. Wiltbank. 2010. Factors affecting fertilisation and early embryo quality in single-and superovulated dairy cattle. Reprod. Fertil. Dev. 22:151-158.

Shahinfar, S., D. Page, J. Guenther, V. Cabrera, P. Fricke, and K. Weigel. 2014. Prediction of insemination outcomes in Holstein dairy cattle using alternative machine learning algorithms. J. Dairy Sci. 97:731-742.
Sing, T., O. Sander, N. Beerenwinkel, and T. Lengauer. 2005. ROCR: Visualizing classifier performance in R. Bioinformatics 21:39403941.

Sorensen, H. T., S. Sabroe, and J. Olsen. 1996. A framework for evaluation of secondary data sources for epidemiological research. Int. J. Epidemiol. 25:435-442.

Strobl, C., A.-L. Boulesteix, A. Zeileis, and T. Hothorn. 2007. Bias in random forest variable importance measures: Illustrations, sources and a solution. BMC Bioinformatics 25:8-28.

Strobl, C., J. Malley, and G. Tutz. 2009. An introduction to recursive partitioning: Rationale, application, and characteristics of classification and regression trees, bagging, and random forests. Psychol. Methods 14:323-348.

Strong, D. M., Y. W. Lee, and R. Y. Wang. 1997. Data quality in context. Commun. ACM 40:103-110.

Sturman, H., E. Oltenacu, and R. Foote. 2000. Importance of inseminating only cows in estrus. Theriogenology 53:1657-1667.

Tayi, G. K., and D. P. Ballou. 1998. Examining data quality. Commun. ACM 41:54-57.

Tibshirani, R. 1996. Regression shrinkage and selection via the lasso. J. R. Stat. Soc. Series B Stat. Methodol. 58:267-288.

Verweij, P. J., and H. C. Van Houwelingen. 1994. Penalized likelihood in Cox regression. Stat. Med. 13:2427-2436.

Wand, Y., and R. Y. Wang. 1996. Anchoring data quality dimensions in ontological foundations. Commun. ACM 39:86-95.

Wang, R. Y., V. C. Storey, and C. P. Firth. 1995. A framework for analysis of data quality research. IEEE Trans. Knowl. Data Eng. 7:623-640.

Wang, R. Y., and D. M. Strong. 1996. Beyond accuracy: What data quality means to data consumers. Inf. Manage. 12:5-33.

Wenz, J. R., and S. K. Giebel. 2012. Retrospective evaluation of health event data recording on 50 dairies using Dairy Comp 305. J. Dairy Sci. 95:4699-4706.

Zwald, N., K. Weigel, Y. Chang, R. Welper, and J. Clay. 2006. Genetic analysis of clinical mastitis data from on-farm management software using threshold models. J. Dairy Sci. 89:330-336.

Zwald, N., K. Weigel, Y. Chang, R. Welper, and J. Clay. 2004. Genetic selection for health traits using producer-recorded data. I. Incidence rates, heritability estimates, and sire breeding values. J. Dairy Sci. 87:4287-4294 